\title{
10 ASSUMED RISK BY VULNERABLE ROADWAY USERS: TRAFFIC RULES TRANSGRESSIONS IN A CAPITAL CITY OF SOUTHWESTER COLOMBIA, 2009
}

doi:10.1136/injuryprev-2012-040590t.10

\footnotetext{
1,2FJ Morales-Quintero, ${ }^{1,2}$ GS Gómez-Salazar, ${ }^{2} \mathrm{FJ}$ Bonilla-Escobar, ${ }^{2} \mathrm{~A}$ Fandiño-Lozada, ${ }^{2} \mathrm{~J}$ Santaella, ${ }^{2}$ MI Gutierrez-Martinez. ${ }^{1}$ School of Medicine, Health Faculty, Universidad del Valle, Cali, Colombia; ${ }^{2}$ CISALVA Institute, Health Faculty, Universidad del Valle, Cali, Colombia
}

Background Traffic rules transgressions are a risk factor for traffic events; their fulfilment reduced on a half the number of deaths and serious injuries.

Aims/Objectives/Purpose To determine the traffic rules infractions on roads by users, in places where traffic events occurred with injuries. Cali, Colombia.

Methods Descriptive study of road traffic videos filmed at sites of occurrence of traffic injury. The sample consisted of 12 videos of $30 \mathrm{~min}$ each, we established users, transgressions of traffic rules and their confidence intervals. The proportions were compared with the Z statistic, with 95\% confidence.

Results/0utcomes There were a total of 13491 road users, distributed as follows: $1.206(8.9 \%)$ pedestrians, $624(4.6 \%)$ cyclists, 3.324 (24.6\%) motorcyclists and $8.337(61.8 \%)$ drivers. The most frequent infraction in drivers was not respecting the stop signal (3.1\%), in motorcyclists transiting in the line designed just for automobiles $(55.2 \%)$, driving without wearing a reflective safety vest $(12.4 \%)$; in cyclists driving without wearing a reflective safety vest $(100 \%)$ and without wearing helmet $(99.2 \%)$. In pedestrians the most frequent infraction was crossing the road on inappropriate place $(77.3 \%)$ not used of the pedestrian bridge $(72.3 \%)$. Significant differences were found between the proportion of 
infractions committed by drivers compared with vulnerable road users $(p<0.001)$.

Significance/Contribution to the Field We observed high proportions of traffic rules transgressions among road users, especially among vulnerable road users, increasing their risk. We recommend studies that address the problems of infractions from the perspective of road user, and the formulation of activities to reduce these behaviours. 